\title{
Near-transform-limited picosecond pulses from a gain-switched InGaAs diode laser with fiber Bragg gratings
}

Cite as: Appl. Phys. Lett. 79, 151 (2001); https://doi.org/10.1063/1.1381412

Submitted: 16 October 2000 . Accepted: 02 May 2001. Published Online: 02 July 2001

D. J. L. Birkin, E. U. Rafailov, W. Sibbett, L. Zhang, Y. Liu, and I. Bennion

\section{ARTICLES YOU MAY BE INTERESTED IN}

$12 \mathrm{~W}$ peak-power 10ps duration optical pulse generation by gain switching of a singletransverse-mode GalnN blue laser diode

Applied Physics Letters 93, 131113 (2008); https://doi.org/10.1063/1.2996025

The effect of cavity length on picosecond pulse generation with highly rf modulated AIGaAs double heterostructure lasers

Applied Physics Letters 39, 860 (1981); https://doi.org/10.1063/1.92612

Observation of ultrashort (<4 ps) gain-switched optical pulses from long-wavelength multiple quantum well lasers

Applied Physics Letters 55, 1273 (1989); https://doi.org/10.1063/1.101630

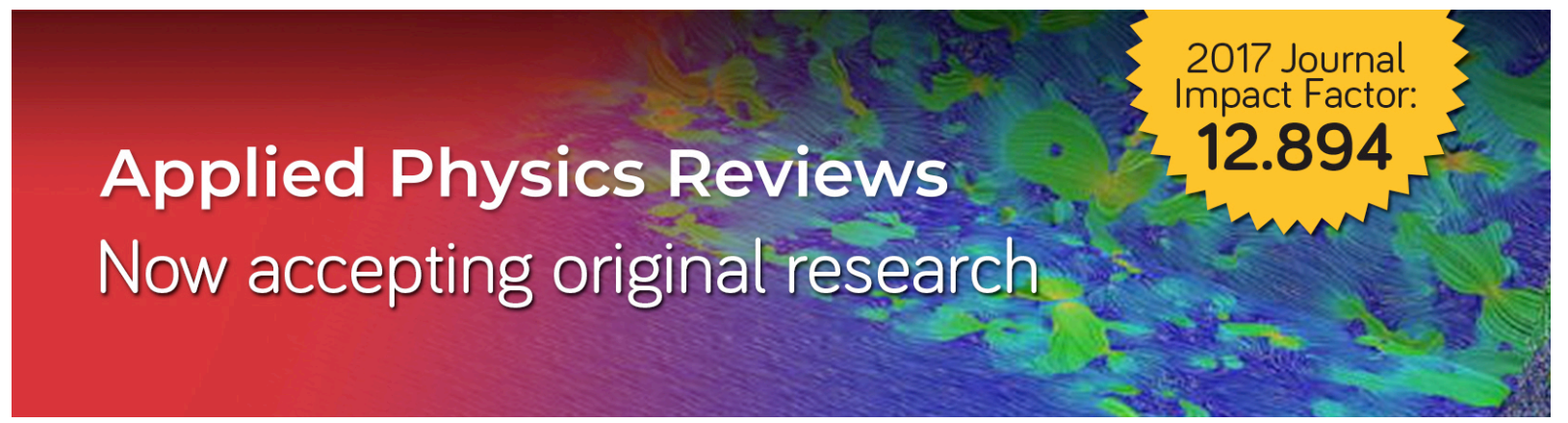




\title{
Near-transform-limited picosecond pulses from a gain-switched InGaAs diode laser with fiber Bragg gratings
}

\author{
D. J. L. Birkin, ${ }^{\text {a) }}$ E. U. Rafailov, and W. Sibbett \\ School of Physics and Astronomy, University of St. Andrews, North Haugh, St. Andrews, Fife KY 16 9SS, \\ United Kingdom \\ L. Zhang, ${ }^{\text {b) }}$ Y. Liu, and I. Bennion \\ Photonics Research Group, Electronics Engineering and Applied Science, Aston University, \\ Birmingham B4 7ET, United Kingdom
}

(Received 16 October 2000; accepted for publication 2 May 2001)

\begin{abstract}
We have generated near-transform-limited picosecond pulses $(\Delta \tau \Delta \nu \approx 0.45)$ from a gain-switched diode laser using periodic and chirped fiber Bragg gratings. This configuration reduced the spectral bandwidth from 11 to $0.08 \mathrm{~nm}$ and the pulse duration was reduced, from 30 to $<18 \mathrm{ps}$. Average and peak powers of 27 and $770 \mathrm{~mW}$, respectively, were obtained. (c) 2001 American Institute of Physics. [DOI: 10.1063/1.1381412]
\end{abstract}

Temporal compression of picosecond diode laser pulses and single-frequency operation is of considerable interest. This is due in part to the expected increase in second harmonic $^{1}$ and optical parametric oscillation ${ }^{2}$ efficiencies that could be realized with the correspondingly increased peak powers, and the reduced spectral bandwidths. With this objective in mind, we used commercial InGaAs/GaAS single-mode ridge waveguide lasers, which had an active stripe width of $3 \mu \mathrm{m}$ and an emission wavelength at $980 \mathrm{~nm}$. The device was AR-coated, $<3 \%$, on the front facet, and HR-coated $>95 \%$ on the rear facet. The typical gainswitched pulse duration obtained from a laser without optical feedback was $\sim 30 \mathrm{ps}$, through the application of a sinusoidal rf modulation frequency, in the range 1.70-2.70 GHz. When the diode laser was forward biased at $140 \mathrm{~mA}$, with a supplementary rf power up to $37 \mathrm{dBm}$, the average and peak optical powers were $\sim 100 \mathrm{~mW}$ and $\sim 1.7 \mathrm{~W}$, respectively. The pulse spectral bandwidth was $\sim 11 \mathrm{~nm}$, with a Fabry-Perot mode bandwidth of $\sim 0.04 \mathrm{~nm}$ and a separation of $0.17 \mathrm{~nm}$ between adjacent modes. ${ }^{3}$ The time-bandwidth product of the gain-switched output was 103 .

For comparative purposes the laser was first buttcoupled to a $50 \mathrm{~cm}$ length of unprocessed fiber of the type from which the fiber gratings would subsequently be fabricated. The fibers were cleaved perpendicular to the core and the ends were not specially treated. For an average optical power of $\sim 70 \mathrm{~mW}$ launched into the optical fiber, the transmitted power was $35 \mathrm{~mW}$, and this was unaffected by the rf power or frequency. It should be noted that the fiber end was simply cleaved and thus the optical coupling was not optimized. The spectral and temporal characteristics of the transmitted pulse were identical to those of the solitary device.

The fiber Bragg gratings (FBGs) that were assessed initially were periodic with gratings of length $6 \mathrm{~mm}$ and period $0.35 \mu \mathrm{m}$, with peak reflectivites ranging from $20 \%$ to $50 \%$. The Bragg grating was situated in the center of a $\sim 50 \mathrm{~cm}$ long optical fiber. The Bragg grating had a design wave-

\footnotetext{
${ }^{a)}$ Electronic mail: djlb@st-andrews.ac.uk

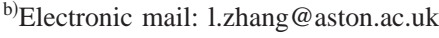

length of $979 \mathrm{~nm}$ with a corresponding full width at half maximum spectral reflectivity of $\sim 0.1 \mathrm{~nm}$. The external cavity formed by the diode laser and fiber Bragg grating had a considerable effect on the characteristics of the pulse transmitted through the optical fiber with the duration and bandwidth becoming $<18 \mathrm{ps}$ and $0.1 \mathrm{~nm}$, respectively. (Measurement of the pulse duration was equipment limited to $18 \mathrm{ps}$.) The optical spectrum was centered at $978.5 \mathrm{~nm}$. Figure 1 illustrates the difference in the spectral characteristics of a gain-switched diode laser for (a) without and (b) with optical feedback from a fiber Bragg grating. The observed spectral and temporal improvement corresponded to a timebandwidth product of 0.56 , representing a reduction of 180 over the product in the absence of optical feedback. Interestingly, the power transmitted through the optical fiber containing the Bragg grating was dependent upon the rf modulation applied to the diode laser. With the rf applied, such that the laser was operating in the picosecond-pulse regime, the transmitted power was $27 \mathrm{~mW}$ whereas the power monitored was just $8 \mathrm{~mW}$ when the rf was not applied. The Bragg grating reflectivity did not affect significantly the transmitted pulse characteristics with the exception of transmitted power.

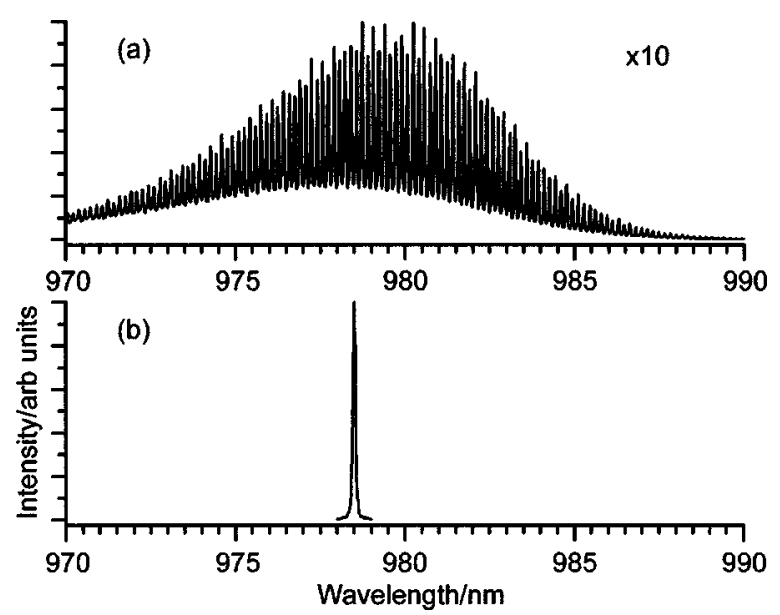

FIG. 1. Spectral characteristics for a gain-switched diode laser for (a) without and (b) with optical feedback from a fiber Bragg grating. 
Given that the Bragg grating reflectivity determined the observed transmitted power, it is reasonable to conclude that an external cavity was formed with the diode chip and the Bragg grating, rather than being a spectral filtering configuration.

It can be seen from Fig. 1(b) that the external cavity formed by the Bragg grating in the optical fiber had a pronounced effect on the spectral characteristic of the laser. The pulse duration was decreased from $30 \mathrm{ps}$ to a minimum of 18 ps, although the pulse was slightly asymmetric. Indeed, if optimum temporal compression could be achieved to produce Fourier-transform-limited pulses then the $11 \mathrm{~nm}$ bandwidth would imply that durations as short as $\sim 150 \mathrm{fs}$ might be obtained. With this objective of temporal pulse compression in mind fiber Bragg gratings were designed and fabricated to compensate for the frequency chirp on the pulses from the diode laser. The magnitude of the frequency chirp in the output of the gain-switched diode laser was obtained from sonogram experiments, ${ }^{4}$ and formed the basis for a design of an optical fiber containing an aperiodic fiber Bragg grating structure, which could accommodate the entire spectral bandwidth of the pulse. Such a grating structure when used in an external cavity configuration would compensate for the group velocity delay present on the chirped pulses. The resultant optical fibers contained aperiodic fiber Bragg gratings that were $6 \mathrm{~mm}$ long with a spectral bandwidth of $10 \mathrm{~nm}$ and a peak reflectivity of $50 \%,{ }^{5}$ and had a design wavelength of $980 \mathrm{~nm}$.

An optical fiber containing an aperiodic fiber Bragg grating was mounted initially with the shorter-period end of the Bragg grating near the emission facet of the diode laser chip. For a laser output power of $\sim 70 \mathrm{~mW}$ the maximum transmitted power was $20 \mathrm{~mW}$. Analogous with the optical fibers containing periodic Bragg gratings, when the rf was turned off spectral filtering of the $\mathrm{cw}$ diode laser output significantly reduced the transmitted power. Illustrated in Fig. 2(a) is the transmitted optical spectrum, centered at $973.5 \mathrm{~nm}$ with a bandwidth of $0.1 \mathrm{~nm}$. The minimum pulse duration was $<22$ ps. The optical fiber was then reversed, such that the longer-period end of the Bragg grating was at the input. In this orientation the optical spectrum was centered at $984 \mathrm{~nm}$ with a bandwidth of $0.08 \mathrm{~nm}$ as shown in Fig. 2(b). The output was more stable when the longer-period end of the Bragg grating was the input and the minimum pulse duration was $<18$ ps with $\Delta \tau \Delta \nu \leqslant 0.45$.

Although spectral and temporal compressions were observed with both types of fiber Bragg grating, the degree of temporal compression was rather poor (factor of $\sim 2$ ) compared with the spectral compression (factor of $\sim 138$ ). An explanation as to why significant temporal compression was not observed with the aperiodic fiber Bragg grating structure can be based on the fact that the grating period closest to the laser was dominating the reflection. This is consistent with

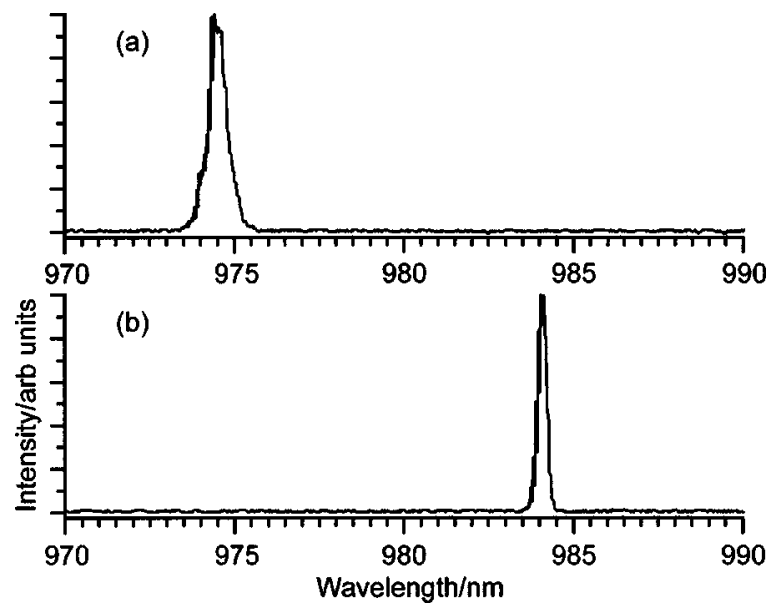

FIG. 2. Spectra of the pulse transmitted through the aperiodic FBG for (a) shorter-period and (b) longer-period as input.

the observation of the two extreme transmitted wavelengths when the optical fiber containing the aperiodic Bragg grating was reversed.

Further improvements to this system will include fusion splicing a fiber pigtailed laser or a lensed fiber to one of the optical fibers containing a fiber Bragg gratings to maximize optical coupling. The transmitted power levels would then be increased by the improved coupling. By using an aperiodic fiber grating with an optical delay it may be possible to obtain pulse compression and tune the wavelength of the transmitted pulse. (An optical delay was not used in this study and so only the two extreme wavelengths were demonstrated.) A theoretic analysis of the results presented earlier is currently ongoing and will be presented at a later date.

In conclusion, we have generated near-transform-limited picosecond pulses from a gain-switched InGaAs diode laser using fiber Bragg gratings in an external cavity configuration. Bandwidth-duration products of $<0.45$ were measured for pulses having durations $<18 \mathrm{ps}$. Peak pulse power levels of $770 \mathrm{~mW}$ were obtained using this laser arrangement.

This work was supported by the UK Engineering and Physical Sciences Research Council which also provided a Ph.D Studentship (D.B.). The authors wish to thank JDS Uniphase (Zurich) for supplying the diode lasers used in this work.

${ }^{1}$ D. J. L. Birkin, E. U. Rafailov, G. S. Sokolovskii, D. T. Reid, W. Sibbett, G. W. Ross, P. G. R. Smith, and D. C. Hanna, Techn. Digest, CLEO'2000, 2000, San Francisco.

${ }^{2}$ A. J. Henderson, P. M. Roper, L. A. Borschowa, and R. D. Mead, Techn. Digest, CLEO'2000, 2000, San Francisco.

${ }^{3}$ E. U. Rafailov, D. J. L. Birkin, E. A. Avrutin, W. E. Sleat, and W. Sibbett, IEE Proc.-J: Optoelectron. 146, 51 (1999).

${ }^{4}$ D. J. L. Birkin, PhD Thesis, St Andrews, 2001 (unpublished).

${ }^{5}$ J. A. R. Williams, I. Bennion, and L. Zhang, IEEE Photonics Technol. Lett. 7, 491 (1995). 